\title{
Morphology and surface reconstructions of m-plane GaN
}

C. D. Lee, ${ }^{1}$ R. M. Feenstra, ${ }^{1}$ J. E. Northrup, ${ }^{2}$ L. Lymperakis, ${ }^{3}$ J. Neugebauer ${ }^{3}$

${ }^{1}$ Department of Physics, Carnegie Mellon University, Pittsburgh, PA 15213

${ }^{2}$ Palo Alto Research Center, 3333 Coyote Hill Road, Palo Alto, CA 94304

${ }^{3}$ Fritz-Haber-Institut der Max-Planck-Gesellschaft, Faradayweg 4-6, D-14195 Berlin, Germany

\begin{abstract}
M-plane $\mathrm{GaN}(1 \overline{1} 00)$ is grown by plasma assisted molecular beam epitaxy on $\mathrm{ZnO}(1 \overline{1} 00)$ substrates. A low-temperature GaN buffer layer is found to be necessary to obtain good structural quality of the films. Well oriented ( $1 \overline{1} 00) \mathrm{GaN}$ films are obtained, with a slate like surface morphology. On the GaN $(1 \overline{1} 00)$ surfaces, reconstructions with symmetry of $\mathrm{c}(2 \times 2)$ and approximate " $4 \times 5$ " are found under $\mathrm{N}$ - and Ga-rich conditions, respectively. We propose a model for Ga-rich conditions with the " $4 \times 5$ " structure consisting of $\geq 2$ monolayers of $\mathrm{Ga}$ terminating the $\mathrm{GaN}$ surface.
\end{abstract}

\section{INTRODUCTION}

Wurtzite GaN heteroepitaxy and surface reconstructions have been extensively studied in the past 5-10 years [1,2]. It is well known that GaN films with (0001) and $(000 \overline{1})$ surface orientations have pyroelectric and piezoelectric properties [3,4], leading to strong electric fields along the crystal c-axis which have useful device applications such as the formation of a twodimensional electron gas near a heterointerface. However these strong electric fields also produce a shift in the wavelength of emission lines and reduced quantum efficiency because of the spatial separation of electrons and holes in quantum wells. Thus, several growth studies of $(1 \overline{1} 00)$ oriented (m-plane) GaN have been performed, since for this orientation the crystal symmetry precludes pyroelectric and piezoelectric effects (at least in the absence of shear stresses in the growth plane) [5-7].

In this work we have used plasma assisted molecular beam epitaxy (PAMBE) to grow GaN films on $\mathrm{ZnO}(1 \overline{1} 00)$ substrates. We find that a low temperature $\left(\sim 500^{\circ} \mathrm{C}\right)$ buffer layer is needed to obtain smooth morphology. Several reconstructions on the GaN $(1 \overline{1} 00)$ surfaces are observed for both Ga- and N-rich conditions. The most dominant of these occurs under Ga-rich conditions and it has an approximate surface unit cell of $4 \times 5$. We propose a model for this structure in which it consists of a contracted and distorted arrangement of a Ga bilayer on the surface.

\section{EXPERIMENTAL}

$\mathrm{ZnO}(1 \overline{1} 00)$ substrates were obtained from Cermet, Inc. The substrates were prepared by mechanical polishing; atomic force microscopy (AFM) revealed many scratches and polishing marks on the substrate surfaces. The MBE growth and subsequent scanning tunneling microscopy (STM) were performed using a system previously described [1,2]. The ZnO substrates were cleaned inside the growth chamber prior to growth simply by heating them to about $550^{\circ} \mathrm{C}$ for $20 \mathrm{~min}$. Growth was initiated by exposing the $\mathrm{ZnO}$ surface simultaneously to 
incident $\mathrm{Ga}$ and $\mathrm{N}$ fluxes. $\mathrm{A} \approx 20 \mathrm{~nm}$ thick $\mathrm{GaN}$ layer is grown at a relatively low temperature of about $500^{\circ} \mathrm{C}$, and subsequent growth was performed at about $700-750^{\circ} \mathrm{C}$. If growth is performed in the absence of the low temperature buffer layer, a very spotty RHEED pattern forms during the initial stages of growth, indicating a rough morphology. Similar spotty RHEED patterns are obtained whenever the $\mathrm{ZnO}$ is exposed to the $\mathrm{Ga}$ beam at a temperature of $>550^{\circ} \mathrm{C}$ (i.e. in the absence of the low temperature buffer), possibly indicating a reaction between $\mathrm{Ga}$ atoms and $\mathrm{O}$ atoms leading to the dissociation of $\mathrm{ZnO}$. Although we do not know the detailed chemical reactions occurring on the substrate, the low temperature $\left(\sim 500^{\circ} \mathrm{C}\right)$ growth certainly results in a significant improvement of surface morphology. During and following the growth the surface is characterized by reflection high energy electron diffraction (RHEED). After the growth the samples were cooled and transferred under ultra high vacuum to an adjoining analysis chamber for STM study.

\section{RESULTS \& DISCUSSION $\underline{\text { Experiment }}$}

Figure 1(a) shows a typical surface morphology for our m-plane GaN films, grown with thicknesses of about $0.5 \mu \mathrm{m}$ grown and generally under similar Ga-rich growth conditions as used for our c-plane growth [8]. RHEED study reveals that the films have the same in-plane orientation as the substrate, i.e. with $\mathrm{GaN}(0001) / / \mathrm{ZnO}(0001)$ and $\mathrm{GaN}(11 \overline{2} 0) / / \mathrm{ZnO}(11 \overline{2} 0)$, thereby demonstrating the $(1 \overline{1} 00)$ orientation of the films. The substrates have an unintentional miscut of several degrees oriented primarily towards the [0001] direction, thus producing the steps seen extending vertically in the image. In Fig 1(a), large flat $\mathrm{GaN}(1 \overline{1} 00)$ terraces are found. The terraces tend to be elongated in the [11 $\overline{2} 0]$ direction, forming a slate like morphology. The surface morphology and the lattice constants obtained from RHEED are similar to that reported by Waltereit et al. [5]. RHEED patterns of the films are streaky, as shown in Fig. 1(b), consistent with the flat terraces observed in the morphology. The RHEED shows a streaky $1 \times 1$ pattern throughout the entire growth sequence when Ga-rich conditions are used. Consistent with our prior c-plane $\mathrm{GaN}$ growth on $\mathrm{SiC}$ [8], growth under $\mathrm{N}$-rich conditions results
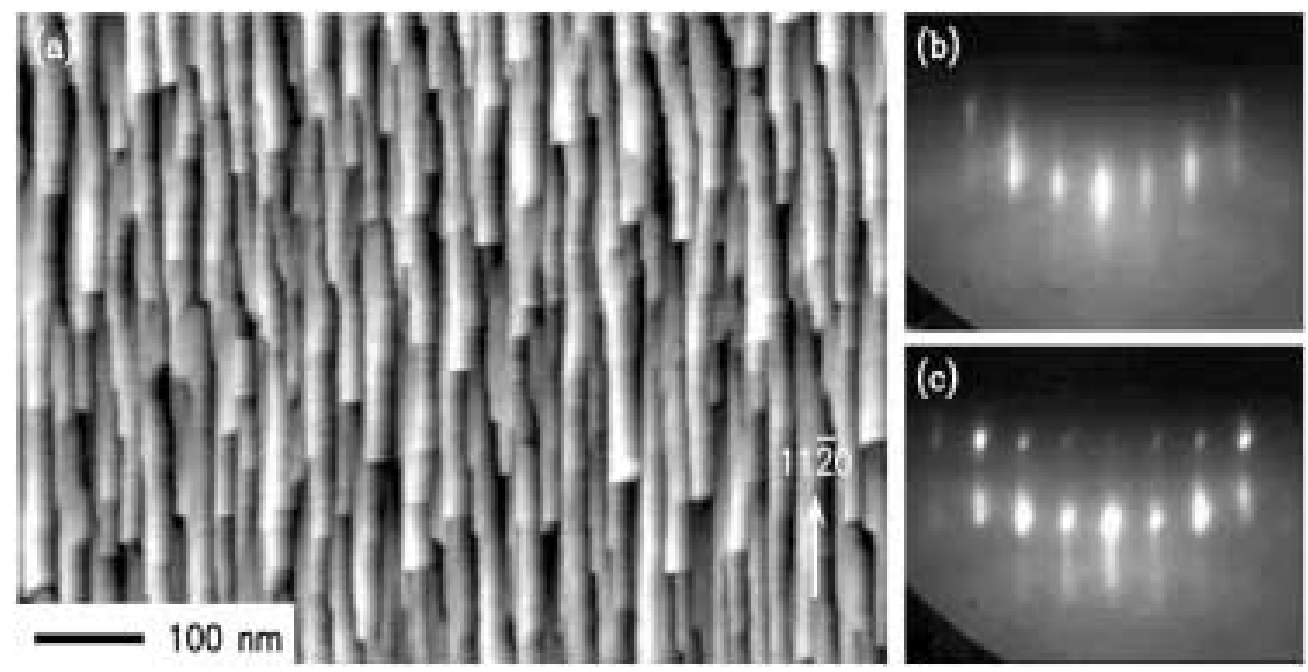

FIG 1 (a) STM image of $\mathrm{GaN}$ film grown on $\mathrm{ZnO} \quad(1 \overline{1} 00)$ substrate. and (c) RHEED patterns (with electron beam along [11 $\overline{2} 0]$ ) acquired under Ga-rich and Nrich conditions, respectively. 

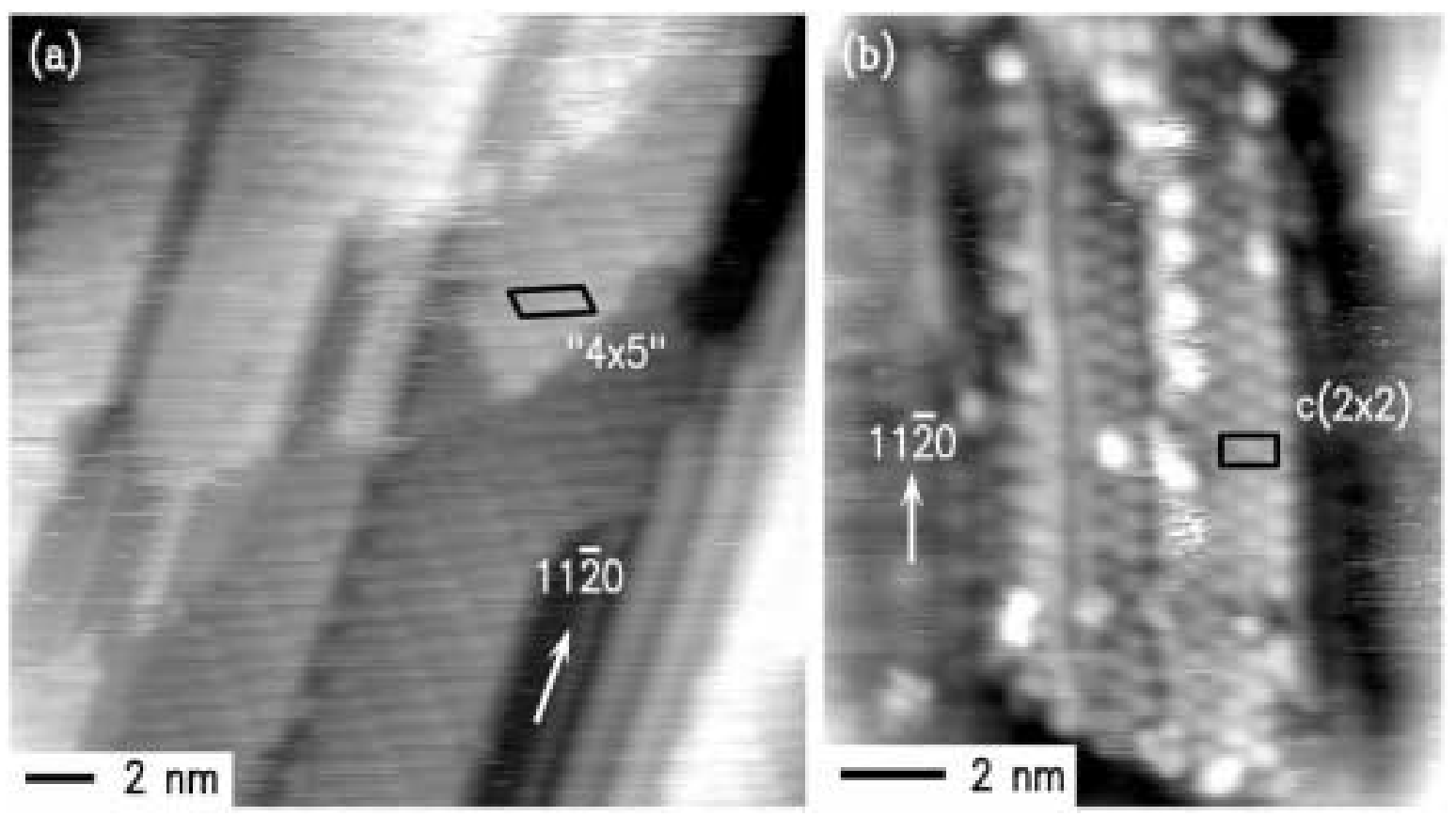

FIG 2 (a) STM image of Ga-rich GaN(1 100$)$ surface. The image was acquired with a sample voltage of $-2.0 \mathrm{~V}$ and is displayed with a gray scale range of $8.5 \AA$. (b) STM image of nitrided surface showing reconstruction with $\mathrm{c}(2 \times 2)$ unit cell. The image was acquired with a sample voltage of $-1.0 \mathrm{~V}$ and is displayed with a gray scale range of $3.0 \AA$.

in spotty RHEED patterns indicative of 3-dimensional growth as shown in Fig. 1(c). For thicker films the RHEED patterns display a clear evidence of facetting, as described elsewhere [7].

We have observed several reconstructions on the $\mathrm{GaN}(1 \overline{1} 00)$ terraces, with our clearest observations being for films grown under Ga-rich conditions as pictured in Fig. 2(a). Terraces extending in the $[11 \overline{2} 0$ ] direction are seen there separated by narrow trenches and/or raised rows of atoms. The triangular shape of step edges seen in Fig. 2(a) are often observed. We note that the elongation along the [11 $\overline{2} 0$ ] direction is the same as recently reported for films grown by metal-organic chemical vapor deposition. [8]. This anisotropic morphology may arise from an anisotropy in the migration lengths of adatoms. A reconstruction of the surface is visible in Fig. 2(a); the slightly brighter rows of atoms seen there extend at angles of $\pm 22^{\circ}$ away from the [11 $\overline{2} 0$ ] direction (these angles are slightly distorted in Fig. 2(a), due to drift). A unit cell is indicated in the image. As discussed in the following section, and described in detail elsewhere [7], the basis vectors for this unit cell are $4 \mathbf{a}+1 \mathbf{c}$ and $-1 \mathbf{a}+5 \mathbf{c}$, where $\mathbf{a}$ and $\mathbf{c}$ span the $1 \times 1$ surface unit cell. The structure thus contains $211 \times 1$ cells. This surface unit cell can be constructed from a $4 \mathbf{a} \times 5 \mathbf{c}$ cell by slightly rotating and shearing it, and so for a shorthand notation we refer to our observed structure as "4×5" (including quotation marks to indicate an approximate symmetry). This surface appears to be metallic since relatively little difference is found between the empty and filled states images, and imaging at low voltages of $\pm 0.1 \mathrm{~V}$ is found to be possible [7].

Figure 2(b) shows an STM image obtained from the surface which had been nitrided for $1 \mathrm{~min}$ following growth. A different reconstruction is observed here than in Fig. 2(a). We find a 
$c(2 \times 2)$ unit cell as indicated in the image. RHEED patterns for this film display weak $2 \times$ streaks for both the [0001] and [11 $\overline{2} 0]$ azimuths. This type of structure has been observed reproducibly on surfaces prepared under N-rich conditions, but we have not investigated in detail its voltage dependence nor the underlying geometric arrangement of atoms.

\section{Theory}

Theoretical studies on $\mathrm{GaN}(1 \overline{1} 00)$ surfaces have been reported in previous studies [7,9]. Initially, the focus was on structural models similar to those found on cubic III-V (110) surfaces, i.e., models which have a $1 \times 1$ surface unit cell and obey electron counting [9]. These structures are characterized by two threefold coordinated surface atoms in each $1 \times 1$ cell. In the stoichiometric case the two atoms are a $\mathrm{Ga}$ and a $\mathrm{N}$ atom, and the surface is stabilized by a charge transfer from the cation to the anion dangling bond and a subsequent rehybridization causing the $\mathrm{N}$ to move slightly outward and the Ga to move slightly inward. This surface, which would be expected to be present following cleavage or cracking, has been found to be energetically favorable over a large range of the thermodynamically allowed chemical potentials. However, when going to rather Ga-rich conditions (i.e., to conditions realized in the experiments described here) structures with excess Ga atoms become more stable [7,9]. These structures are formed from the stoichiometric structure by (i) replacing the $\mathrm{N}$ surface atom in the $1 \times 1$ surface cell by a Ga atom and/or (ii) by adding two monolayers (ML, defined as one atom per $1 \times 1$ cell) $\mathrm{Ga}$ on top of this surface. Since the Ga-Ga spacing in the Ga adlayer is slightly larger than in $\mathrm{Ga}$ bulk, an additional lowering of the surface energy might be achieved by compressing the Ga atoms in the top layer such that their density approaches something close to that of Ga bulk. In Ref. [7] we have briefly discussed the energetics of such a structure without giving details regarding the calculational approach, geometry or construction of the surface. Below we provide this additional information.

To set up the structure we combined the structural information from the STM data regarding size, symmetry and orientation of the surface unit cell and with the energetical considerations from the ab initio calculations. Specifically, we constructed a surface which has the experimentally observed " $4 \times 5$ " unit cell and in which the rows of Ga atoms in the adlayer follow the outline of this cell. The structure is pictured in Fig. 3. It consists of the stoichiometric surface containing $21 \mathrm{Ga}-\mathrm{N}$ dimers, on top of which is placed a compressed $\mathrm{Ga}$ adlayer consisting of 45 atoms (see Fig. 3(a)). The adlayer thus corresponds to a Ga coverage of 45/21=2.14 ML.

A direct calculation of this structure using the same pseudopotentials (LDA Troullier Martins pseudopotentials with $\mathrm{Ga} 3 d$ in the valence), plane wave energy cutoff (60 Ry), and slab thickness (4 layers) as for the $1 \times 1$ structures [9] turned out to be computationally too expensive. We have therefore performed careful convergence checks to identify a parameter set which minimizes the computational effort but still allows to obtain accurate surface geometries and energies. Based on these results we found that using PBE-GGA Troullier Martins pseudopotentials where the $\mathrm{Ga} 3 d$ orbitals are treated in the non-linear core approximation, an energy cutoff of $30 \mathrm{Ry}$ and a slab thickness of $2 \mathrm{GaN}$ layers plus the Ga adlayer are sufficient to describe the surface energy with an accuracy of better than $0.005 \mathrm{eV} / \AA^{2}$, and the surface geometry better than $0.027 \AA$, for Ga-rich surface structures. We note that e.g. for stoichiometric surface structures significantly larger error bars result. To be more specific, let us consider the 

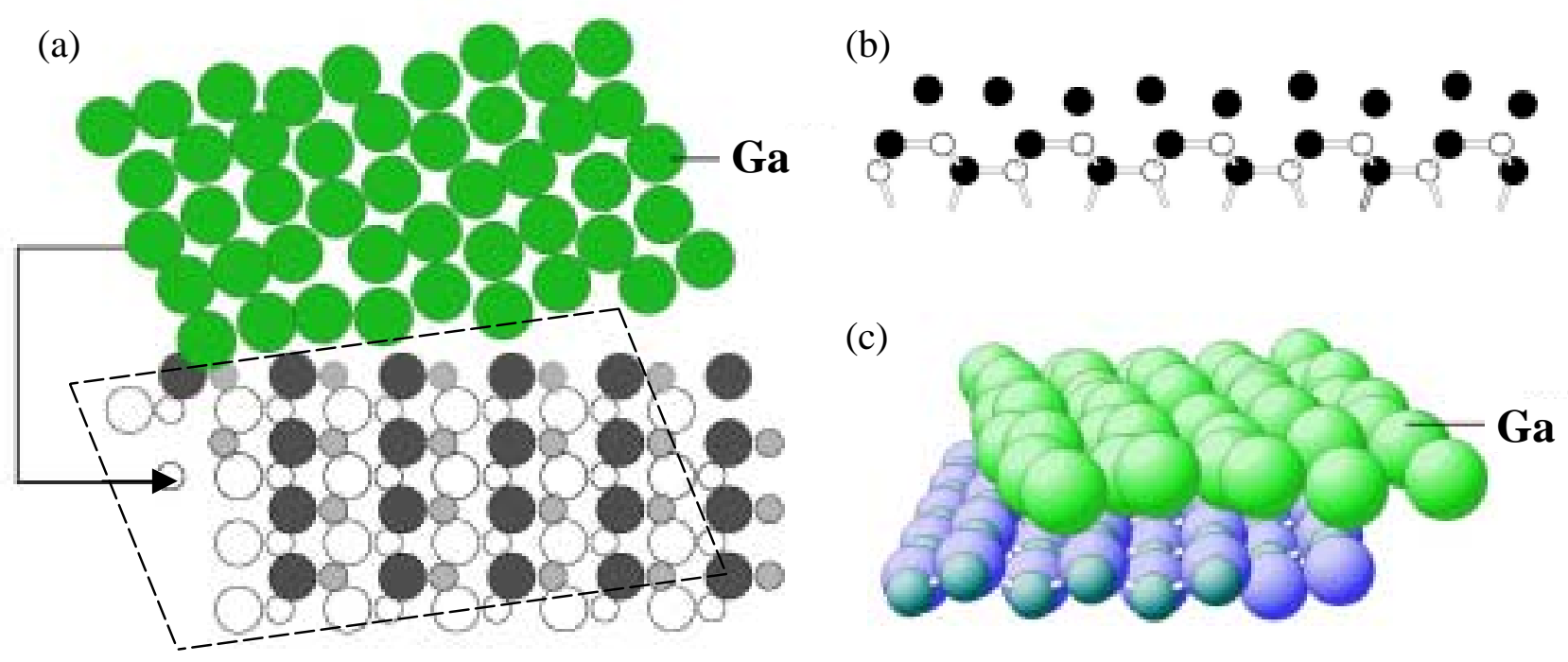

FIG 3 (color) Schematic view of the reconstructed and compressed Ga adlayer GaN(1 $\overline{1} 00)$ surface. (a) top view with " $4 \times 5$ " cell shown by the dashed line, (b) side view and (c) perspective view. In (a), large black or small gray filled circles mark the Ga or $\mathrm{N}$ atoms, respectively, in the top surface layer. The large green (or grey, if not viewed in color) filled circles mark the positions of the Ga atoms in the Ga adlayer, as indicated.

convergence of the surface energy with respect to the slab thickness for the stoichimetric (SD) and the adlayer $(\mathrm{SD}+2)$ structure (SD indicates here a single $\mathrm{GaN}$ dimer, +2 stands for adding 2 $\mathrm{ML}$ of $\mathrm{Ga}$ ). We find that the energy of the stoichiometric surface significantly increases when going from a two to a four layer slab (by more than $0.025 \mathrm{eV} / \AA^{2}$ ) while it changes less than $0.005 \mathrm{eV} / \AA^{2}$ for the adlayer structure. An analysis showed that the origin of the different convergence behavior lies in the presence/absence of nitrogen dangling bond states. The presence of $\mathrm{N}$ dangling bond states causes (as described above) charge transfer, rehybridization and eventually a rather strong atomic relaxation of the $\mathrm{N}$ bond. Since the $\mathrm{N}$ forms stiff bonds with the neighboring $\mathrm{Ga}$ atoms this effect causes considerable strain in the lattice which decays only slowly with increasing slab thickness. On the other hand, adding a $\mathrm{Ga}$ adlayer prevents the formation of $\mathrm{N}$ dangling bonds and the $\mathrm{N}$ atoms stay close to their bulk positions. In this case strain effects in the GaN layer turn out to be negligible.

Using the above described parameter set we have performed a geometry optimization for the structure. To destroy the initially high symmetry of the surface we adsorbed a single adatom and removed it after performing a few atomic steps. The resulting equilibrium geometry is shown in Figs. 3(a-c). As can be seen the Ga adlayer buckles and forms a low symmetry structure. The underlying Ga-N layer, however, is virtually not affected and the atoms are close to their corresponding bulk positions. A closer analysis showed that the low symmetry of the Ga adlayer and the large buckling $(\sim 0.8 \AA)$ is related to the fact that the Ga atoms in the Ga adlayer are out of registry to the underlying Ga-N surface layer.

The out of registry arrangement of the Ga adlayer also affects the stability of this surface: While the compression of the $\mathrm{Ga}$ adlayer is expected to strengthen the Ga-Ga bonds and thus the stability of the surface, the out of registry arrangement weakens the Ga-N bonds. Our total energy calculations show that the two effects almost cancel each other - the energy of the compressed Ga-adlayer structure under very Ga-rich conditions is practically the same as the 
energy of the uncompressed $2 \mathrm{ML} \mathrm{Ga}$ adlayer structures considered previously [7]. It is possible that further optimization of the geometry (i.e. considering other arrangements and/or other densities of the adlayer) may produce a reduction in energy. In any case, judging from the experimental STM images, the arrangement of the adlayer must indeed be a rather complicated one. The investigation described here establishes the energetic feasibility of such structures containing $>2 \mathrm{ML}$ of $\mathrm{Ga}$, and further work is required to definitively determine the complete structural arrangement.

\section{CONCLUSION}

In conclusion, we have grown (1 $\overline{1} 00)$ oriented $\mathrm{GaN}$ films on $\mathrm{ZnO}$. Under Ga rich conditions a " $4 \times 5$ " reconstruction is observed. Experimentally this structure appears to be metallic, indicating that it consists of an adlayer of $\mathrm{Ga}$ on the surface, in analogy with the known structures of $(000 \overline{1})$ and (0001) surfaces [1,2]. Theoretical computations indicate that $\geq 2$ monolayers of Ga terminating the $\mathrm{GaN}$ crystal is energetically favorable, under Ga-rich conditions. We have investigated in detail a distorted, compressed adlayer structure containing 2.14 ML of Ga, and find that its energy is nearly the same as that of the uncompressed 2 ML adlayer.

\section{ACKNOWLEDGMENTS}

This work has been supported by the Office of Naval Research under grant N00014-96-1-0214 (program monitor, C. Wood), the Deutsche Forschungsgemeinschaft SFB "296", and the EU TMR program IPAM.

\section{REFERENCES}

[1] A. R. Smith, R. M. Feenstra, D. W. Greve, J. Neugebauer, J. E. Northrup, Phys. Rev. Lett. 79, 3934 (1997).

[2] A. R. Smith, R. M. Feenstra, D. W. Greve, M.-S. Shin, M. Skowronski, J. Neugebauer, J. E. Northrup, Surf. Sci. 423, 70 (1999).

[3] A. Bykhovski, B. Gelmont, and M. Shur, J. Appl. Phys. 74, 6734 (1993).

[4] F. Bernardini, V. Fiorentini, and D. Vanderbilt, Phys Rev. B 56, R10024 (1997).

[5] P. Waltereit, O. Brandt, M. Ramsteiner, A. Trampert, H. T. Grahn, J. Menniger, M. Reiche, R. Uecker, P. Reiche, and K. H. Ploog, Phys. Stat. Sol. (a) 180, 133 (2000).

[6] C. Q. Chen, M. E. Gaevski, W. H. Sun, E. Kuokstis, J. P. Zhang, R. S. Q. Fareed, H.M. Wang, J. W. Yang, G. Simin, M. A. Khan, H.-P. Maruska, D. W. Hill, M. M. C. Chou, and B. Chai, Appl. Phys. Lett. 81, 3194 (2002).

[7] C. D. Lee, R. M. Feenstra, J. E. Northrup, L. Lymperakis, and J. Neugebauer, submitted to Appl. Phys. Lett.

[8] C. D. Lee, V. Ramachandran, A. Sagar, R. M. Feenstra, D. W. Greve, W. L. Sarney, L. Salamanca-Riba, D. C. Look, S. Bai, W. J. Choyke, and R. P. Devaty, J. Electron. Mater. 30, 162 (2001).

[9] J. E. Northrup and J. E. Neugebauer, Phys. Rev. B 53, R10477 (1996). 